\title{
Effective Learning Rate Adjustment of EASI Algorithm Based on the Fuzzy Neural Network
}

\author{
Deng-Ao Li*, Yan-Fei Bai, Ju-Min Zhao \\ College of Information Engineering, Taiyuan University of Technology, 030024, Taiyuan, China. \\ * Corresponding author. Tel: 0351-3176507; email: lidengao@tyut.edu.cn \\ Manuscript submitted March 30, 2016; accepted July 29, 2016. \\ doi: $10.17706 /$ jcp.12.6.579-590
}

\begin{abstract}
The behavior of the classic algorithm for blind source separation is reviewed for a fixed step-size. The inherent contradiction of the classic equivariant adaptive source separation via independence algorithm (EASI) make it very difficult to balance convergence speed and the steady-state misadjustment error. In this paper, In response to the above contradiction, a Fuzzy Neural Network(FNN)-based learning rate adjustment method is proposed for EASI algorithm, and a simple decision-making method is introduced for how the learning rate should be applied in the current time slot. Signals separation process adopt the different learning rate according to the characteristics of the separate degree. Therefore it can improve convergence speed and reduce the misadjustment error in the steady state simultaneously. Extensive simulations confirm the theoretical analysis and show the proposed approach is superior to other EASI algorithms.
\end{abstract}

Key words: Adaptive step-size control, fuzzy neural network, EASI algorithm, blind source separation.

\section{Introduction}

The process of estimating the source signals only by the observed signals received from the sensors without knowing any prior knowledge of the source signals and the transport channel is called the blind source separation [1]. It has important research value in the area of signal process, like biomedical signals, image and antenna array signals. The majority of BSS algorithms can be categorized into either block-based or sequential(on-line) techniques. People have developed a number of mature and effective method for adaptive learning for the mixing signals. Online blind source separation usually has three techniques: Principal Component Analysis (SCA), a non-negative matrix factorization (NMF) and independent component analysis (ICA). Among which, ICA is the mainstream approach [2]. It mainly includes Stochastic gradient algorithms (SGA)[3], natural gradient algorithm(NGA)[4], EASI and so on. These algorithms have a solid theoretical foundation and effective separation effect. All of them can be classified into the LMS algorithm. Such algorithms require to choose a good step at the right time. Step-size determines the convergence speed and steady-state performance of the algorithm. The two indexes often contradict each other. Specifically, the smaller the step-size, the smaller steady-state error and the slower convergence speed, Conversely, the bigger learning rate, the faster convergence rate and the worse stability conditions. When the signals to be separated are in the initial stage or in a situation of mutation, we hope choose a stride step so that we can obtain the faster convergence speed and the better tracking ability in a time-varying channel. In the late stages of separation, we are inclined to choose the smaller one to achieve small steady-state error.

As opposed to these previously mentioned methods, this paper adopts a Fuzzy Neural Network (FNN) to 
find a suitable learning rate for BSS, and also extends its performance to save running time, making the system more flexible in adapting to the online learning requirement.

This paper is organized as follows. Section 2 presents an overview of the problem of BSS. Section 3 describes the conventional EASI algorithm. Section 4 presents the proposed method for learning rate adjustment. Section 5 presents experiment results. Section 6 of this paper contains the conclusion.

\section{The On-line BSS Algorithm}

Suppose $n$ unknown statically in dependent zero mean source signals, passing through an unknown mixing channel, such that $n$ mixed signals, $x_{1}(\mathrm{t}), \ldots x_{n}(\mathrm{t}), \mathrm{x} \in \mathrm{R}^{n}$, are therefore observed. The relation between the observed signals and the source signals can be expressed as:

$$
\mathbf{x}(t)=\mathbf{A s}(t)+\mathbf{n}(t)
$$

where $\mathbf{A} \in \mathbf{R}^{n \times n}$ represents the mixing matrix which reflects the transmission characteristics of the channel and is required full rank. $\mathbf{n}(t)$ is the possible contaminating noise vector. In this paper, we assume $m=n$ and don't take the channel convolution or noise interference into consideration, so we can rewrite the equation (1) as the following form:

$$
\mathbf{x}=\mathbf{A s}
$$

If the source signal and the mixing channel are unknown, we can recover the source signals from the observed signals by the following linear transformation:

$$
\mathbf{y}(t)=\mathbf{W x}(t)
$$

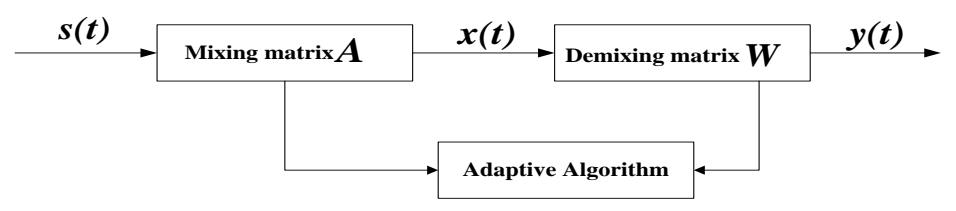

Fig. 1. Model for BSS.

The key aspect of BSS is to seek the optimal separation matrix to make the output component as independent as possible. That is to say, the mutual information of the output signals is minimized. The mutual information is defined as

$$
I(\mathbf{W})=D\left[p(\mathbf{y}, \mathbf{W}) \| \prod_{i=1}^{N} p_{i}\left(\mathbf{y}_{\mathbf{i}}, \mathbf{W}\right)\right]=\int p(\mathbf{y}, \mathbf{W}) \log \frac{p(\mathbf{y}, \mathbf{W})}{\prod_{i=1}^{N} p_{i}\left(\mathbf{y}_{\mathbf{i}}, \mathbf{W}\right)} d \mathbf{y}
$$

$I(\mathbf{W})$ is non-negative, If and only if the output components are independent mutually, namely, $I(W)=0$, only when

$$
\boldsymbol{W}=\boldsymbol{\Lambda} P \boldsymbol{A}^{-1}
$$

where $\boldsymbol{\Lambda}$ is a nonsingular diagonal matrix and $\mathrm{P}$ is a permutation matrix. $\mathbf{A}^{-1}$ is the inverse matrix of $\mathbf{A}$. According to(2),(3)and(5), we can obtain that:

$$
\mathbf{y}=\mathbf{W x}=\mathbf{\Lambda P s}
$$

When the observed signals are separated completely, the result of the BSS has the uncertainty for the magnitude (including phase) and the priorities. However, in practical applications, as long as the shape of the waveform retains unchanged, the two uncertainty are acceptable. 


\section{The Conventional EASI Algorithm}

Using the relative gradient, the conventional EASI [5] and normalized EASI [6] are given in [4], [6] by

$$
\begin{aligned}
\mathbf{W}(k+1) & =\mathbf{W}(k)-\mu\left[\mathbf{y}(k) \mathbf{y}^{T}(k)-\mathbf{I}+g(\mathbf{y}(k)) \mathbf{y}^{T}(k)-\mathbf{y}(k) g^{T}(\mathbf{y}(k))\right] \mathbf{W}(k) \\
\mathbf{W}(k+1) & =\mathbf{W}(k)-\mu\left[\frac{\mathbf{y}(k) \mathbf{y}^{T}(k)-\mathbf{I}}{\mathbf{I}+\mu \mathbf{y}^{T}(k) \mathbf{y}(k)}+\frac{g(\mathbf{y}(k)) \mathbf{y}^{T}(k)-\mathbf{y}(k) g^{T}(\mathbf{y}(k))}{1+\mu \mid \mathbf{y}^{T}(k) g(\mathbf{y}(k))}\right] \mathbf{W}(k)
\end{aligned}
$$

The disadvantages of traditional EASI algorithm is relatively slow convergence speed, and is great impacted by mixing matrix A. In non-stationary environments, algorithms need to have a large step-size to quicken the convergence speed in order to keep up with the change of signals. From the above two equations, we can infer that they have to adopt small fixed step-size to obtain an acceptable separating performance. Thus they cannot reach the combination of fast convergence speed and good separating performance. So a adaptive gradient variable step-size EASI algorithm had been proposed [7] by as follows:

$$
\left\{\begin{array}{l}
\mathbf{W}(k+1)=\mathbf{W}(k)-\mu(k)\left[\mathbf{y}(k) \mathbf{y}^{T}(k)-\mathbf{I}+g(\mathbf{y}(k)) \mathbf{y}^{T}(k)-\mathbf{y}(k) g^{T}(\mathbf{y}(k))\right] \mathbf{W}(k) \\
\mu(k)=\mu(k-1)-\left.\rho \nabla_{\mu} \mathbf{J}(k)\right|_{\mu=\mu(k-1)}
\end{array}\right.
$$

where

$$
\begin{gathered}
\left.\nabla_{\mu} \mathbf{J}(k)\right|_{\mu=\mu(k-1)}=<\frac{\partial \mathbf{J}(k)}{\partial \mathbf{W}(k)}, \frac{\partial \mathbf{W}(k)}{\partial \mu(k-1)}>=\operatorname{tr}\left(\frac{\partial \mathbf{J}(k)}{\partial \mathbf{W}(k)^{T}} \times \frac{\partial \mathbf{W}(k)}{\partial \mu(k-1)}\right) \\
\frac{\partial \mathbf{J}(k)}{\partial \mathbf{W}(k)}=\left[\mathbf{y}(k) \mathbf{y}^{T}(k)-\mathbf{I}+g(\mathbf{y}(k)) \mathbf{y}^{T}(k)-\mathbf{y}(k) g^{T}(\mathbf{y}(k))\right] \mathbf{W}(k)
\end{gathered}
$$

Through computer simulations, this numerical stability of the adaptive gradient variable step-size EASI algorithm is poor and the algorithm is particularly sensitive to the choice of parameter $\rho$. Its disadvantage is as follows: when initial step-size $\mu(0)$ is very small, the algorithm has faster convergence speed and better separating performance than the original algorithms. But with the increase of $\mu(0)$, the algorithm's misadjustment error in the steady state rises faster than convergence speed does. It is regrettable that the algorithm even shows the convergence speed is slower than that of the original algorithms and the misadjustment error is higher than that of the algorithms when $\mu(0)$ is a bit larger. The case completely confirms that the algorithm is not perfect and $\mu(0)$ has to be very small. So the algorithm can not realize the optimal balance between the convergence speed and the misadjustment error [8]. Therefore, we propose phased learning rate EASI algorithm to solve the problem better.

\section{The Learning Rate Adjustment Using the Fuzzy Neural Network}

\subsection{Dependence Measure}

The purpose of BSS is to find the optimal matrix $\mathbf{W}_{o p t}$ to satisfy the smallest independence between output component $\mathbf{y}_{i}$ and $\mathbf{y}_{j} \cdot \mathbf{y}_{i}$ and $\mathbf{y}_{j}$ are independent of each other is equivalent to any metric function of $f_{1}$ and $f_{2}$ [9], that is

$$
E\left\{f_{1}\left(y_{i}\right) f_{2}\left(y_{j}\right)\right\}-E\left\{f_{1}\left(y_{i}\right)\right\} E\left\{f_{2}\left(y_{j}\right)\right\}=0, \forall i \neq j,
$$

As long as $f_{1}\left(y_{i}\right)$ and $f_{2}\left(y_{j}\right)$ are not related to each other, $\mathbf{y}_{i}$ and $\mathbf{y}_{j}$ can be determined independently of each other. Therefore, second-order correlation coefficient is used to measure the independence of signals 
[10]. The second-order correlation between $\mathbf{y}_{i}$ and $\mathbf{y}_{j}$ is defined as

$$
r_{i j}=\frac{C_{i j}}{\sqrt{C_{i i} C_{i j}}}=\frac{\operatorname{cov}\left[y_{i}(k), y_{j}(k)\right]}{\sqrt{\operatorname{cov}\left[y_{i}(k)\right] \operatorname{cov}\left[y_{j}(k)\right]}} \quad i, j=1, \ldots, k \text { and } i \neq j
$$

where

$$
\begin{aligned}
\operatorname{cov}[x(k), y(k)]= & E\left\{\left[x(k)-\bar{m}_{x}\right]\left[y(k)-\bar{m}_{y}\right]\right\} \\
\operatorname{cov}[x(k)] & =E\left\{\left[x(k)-\bar{m}_{x}\right]^{2}\right\} \\
\bar{m}_{x} & =E\{x(k)\}
\end{aligned}
$$

$0 \leq\left|r_{i j}\right| \leq 1$ is the correlation between $\mathbf{y}_{i}$ and $\mathbf{y}_{j}$. When $r_{i j}=0, \mathbf{y}_{i}$ and $\mathbf{y}_{j}$ can be considered independently of each other. Let $D_{i}(k)$ denote the second-order correlation measurement of $\mathbf{y}_{i}$ relative to all the other output components $\mathbf{y}_{j}, j=1, \ldots, m$, where $i \neq j$, hence the following can be obtained:

$$
D_{i}(k)=\sqrt{\frac{1}{k-1}} \sum_{j=1 \ldots k, i \neq j} r_{i j}^{2}
$$

In order to describe the overall state of all signals separated, and defined as

$$
D(k)=\max _{\forall i}\left\{D_{i}(k)\right\}
$$

Clearly, the second-order correlation $D(k)$ measure can describe the dependence of the output component with the others. In this context, the following basic can be described.

1) If $D(k)$ is sufficiently low, then the output component is almost independent of all the other output components, i.e., the separation state of from the others is desirable.

2) If $D(k)$ is not low enough, then the output component is correlated with at least one other output component, i.e., the separation state of is not desirable.

3) If $D(k)$ is too high, then the is correlated strongly with the other outputs, i.e., the separation state of is horrible.

In order to obtain good separation results. The decision-making rule(s) of reducing $D(k)$ need to be made. In the following section, a detailed description of the decision-making process is given.

\subsection{The Fuzzy Neural Network for Learning Rate Adjustment}

In this section we will present a simple fuzzy logic system implemented by using a multilayer feedforward neural network. A schematic diagram of the proposed fuzzy neural network (FNN) structure with $n$ input variables, and $i$ output nodes is shown in Fig. 2. The system consists of four layers. Nodes in layer one are input nodes which represent input linguistic variables. Nodes in layer two are membership nodes which act like membership functions. Each membership node is responsible for mapping an input linguistic variable into a possibility distribution for that variable. The rule nodes reside in layer three. Taken together, all the layer three nodes form a fuzzy rule base. Layer four, the last layer, contains the output variable nodes. The links between the membership nodes and the rule nodes are the antecedent links and those between the rule nodes and the output nodes are the consequence links. For each rule node, there is at most one antecedent link from a membership node of a linguistic variable. Hence all consequence links are fully connected to the output nodes and interpreted directly as the strength of the output action. In this way, the consequence of a 
rule is simply the product of the rule node output, which is the firing strength of the fuzzy rule and the consequence link. Thus, the overall net output is treated as a linear combination of the consequences of all rules instead of the complex composition, a rule of inference and the defuzzification process [11], [12].

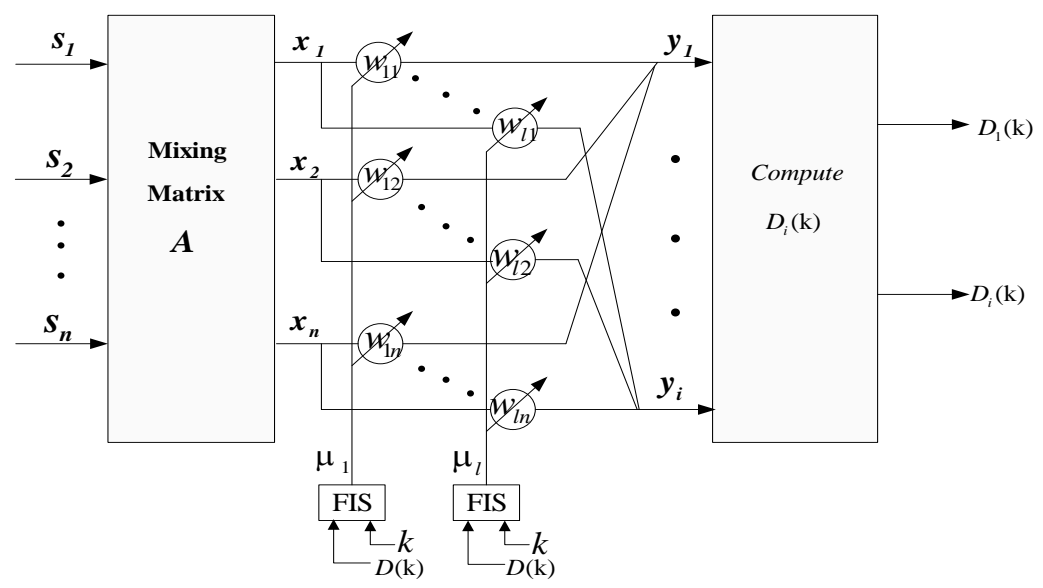

Fig. 2. Blind source separation system using a fuzzy-neural network.

Table 1. Fuzzy Inference Rules

\begin{tabular}{l|l|l}
\hline & Small & Big \\
\hline Small & $I I$ & $I V$ \\
\hline Mid & $I I$ & $I I I$ \\
\hline Big & $I$ & $I$ \\
\hline
\end{tabular}

$\mathbf{W}$ is the $l \times m$ matrix, where $l=m, m-1, \ldots, n$ is dynamically varied Fig. 1 shows our fuzzy-neural network for the EASI in this case, where $w_{i j}$ are the synapse weights of $l \times m$ the demixing or separating matrix $\mathbf{W}$, and $y(k)=\mathbf{W} x(k)$ is the $l \times 1$ output vector, while the weights associated with $y_{i}(k)=\sum_{j=1}^{m} w_{i j} x_{j}(k)$ are adjusted by the learning rate, while $\mu_{i}$ is determined by the FIS. The separation of state of the two signals can be described using $D(k)$. But $D(k)$ change slowly. In order to make the algorithm more sensitive to the learning rate, the entire iterative process requires a finer division. Through simulation experiments carried out to determine the scope of $D(k)$, combined with the number of iterations $k$. Let $k$ and $D_{i}(k)$ be the fuzzy variables which represent the fuzzy membership degrees corresponding to $k$ and $D_{i}(k)$ respectively. We need to adjust the learning rates finely at the tracking stage, so we divide the number of iterations $k$ into two subsets called Small and Big when and take values in the supports $k \leq k_{0}$, and $k>k_{0}$, respectively. We refer to $D_{i}(k)$ as Small, Mid (i.e., Middle), and Big, when and are located at the interval $[0,0.1),[0.1,0.2)$, and $[0.2,1]$, respectively. And the FIS consists of two inputs, respectively, having three and two linguistic values and one output with four linguistic values, it has $3 \times 2=6$ fuzzy inference rules, as shown in Table 1 . These rules can be described as follows:

FIS: If $k$ is $F V_{l}(p)$ and $D_{i}(k)$ is $F V_{2}(q)$, then $\mu_{i}(k)$ is $F V_{3}(r)$

For $i=1, \ldots, l$, where linguistic variables $F V_{1}(p)$ take Small or Big, and linguistic variables $F V_{2}(p)$ take 
Small, Mid, or Big; while $F V_{3}(r)$ takes I, II, III or IV; see Table 1.

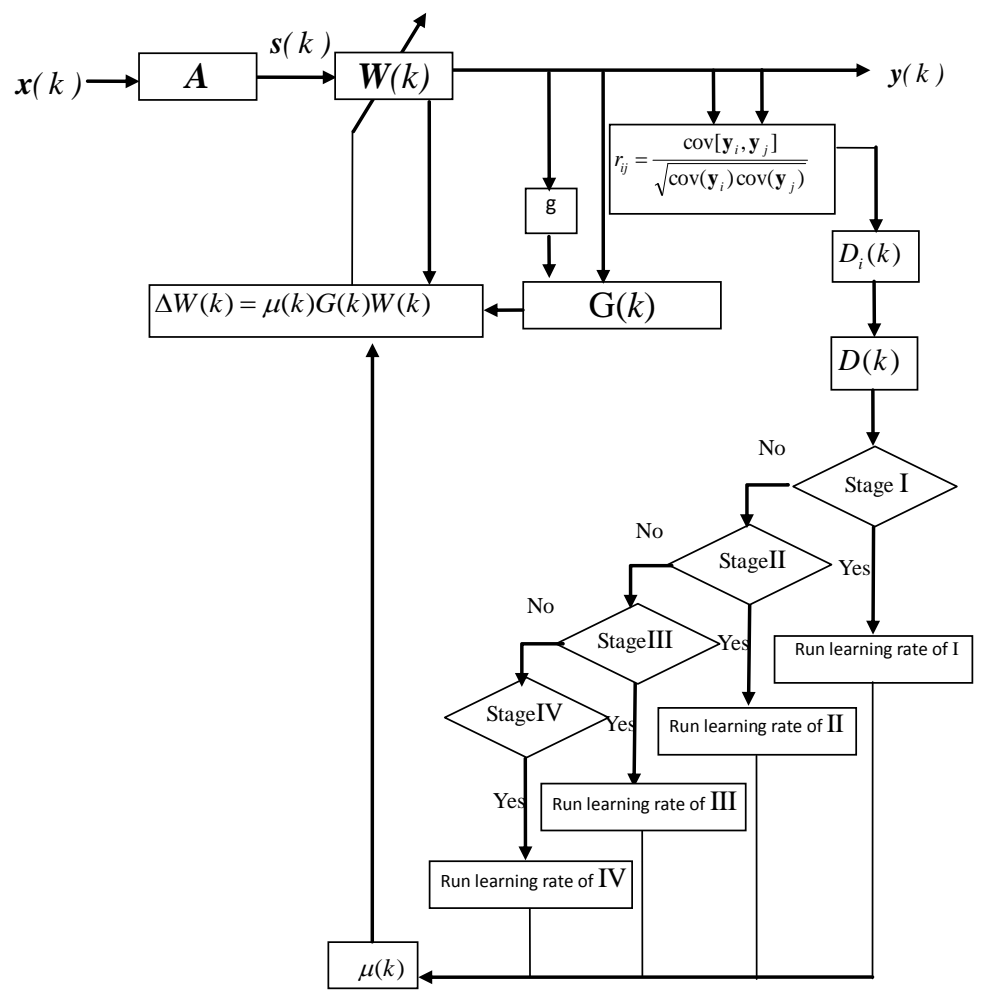

Fig. 3. Concept of the adaptive BSS algorithm with the FNN-based self-adjusting step-size.

In the adaptive separation process, the different learning rates are used at the different rules and it can effectively solve the contradiction between convergence rate and steady-state error [13]-[15].

Rule I: when $D(k)$ is located at the interval $0.2 \leq D(k) \leq 1$, the separation state is poor ,signal separation just begin, and each signal is strong dependence. In this case the larger step size needed in order to accelerate the separation of the signal. So, at this stage in a certain number of iterations, we can choose a big fixed step-size as follows:

$$
\mu_{i}(k)=\mu_{0}
$$

where $\mu_{0}$ is a constant.

Rule II: When iterations $\mathrm{k}$ and $D(k)$ are located at the interval $k<k_{0}$ and $0 \leq D(k)<0.2$, respectively, the moderate separation state is reached and signal separation has just begun. But the signals have been better separation. In practice, this situation is relatively rare. At this point, the signal has been partially separated. Signal separation has just begun and has the smaller dependency degrees. At this stage, we can choose a small step-size adaptive tracking. Step-size selection is as follows:

$$
\mu_{i}(k)=f(D(k))
$$

where $f$ is a nonlinear function(the simulation section will explain it).

Rule III: When iterations $\mathrm{k}$ and $D(k)$ are located at the interval $k>k_{0}$ and $0.1 \leq D(k)<0.2$, respectively, the moderate separation state is reached and signal separation run for a number of times. At this point, part of the signal has been isolated and need for accelerating the capture unseparated. This stage should track the signal and accelerated to capture the unseparated signals. Step-size selection is as follows: 


$$
\mu_{i}(k)=\beta\left(\frac{1}{1+e^{-\alpha D(k)}}-0.5\right)
$$

where $\alpha, \beta$ is a constant.

Rule $I V$ : When iterations $k$ and $D(k)$ are located at the interval $k>k_{0}$ and $0 \leq D(k)<0.1$, respectively, the separation state is quite good. At this point, signal has been basically isolated. In order to pursue better separation that all signal components are captured and the step-size should be taken relatively small value. We should minimize the impact between the various components to improve the quality of the signal recovery, to achieve better separation of source signals. Step-size selection is as follows:

$$
\mu_{i}(k)=\mu_{0} e^{-k_{d}\left(k-k_{0}\right)}
$$

where $u_{0}, k_{0}$ is a constant.

The FNN-based learning rate EASI algorithm flowchart is as follows:

\section{Simulation}

\subsection{Five Source Signals Separation}

In order to verify the effectiveness of the FNN-based learning rate EASI algorithm proposed in this paper, we consider the source signals.

$$
\boldsymbol{s}(t)=\left[\begin{array}{l}
\operatorname{sgn}(\cos (2 \pi 155 t)) \\
\sin (2 \pi 800 t) \\
\sin (2 \pi 300 t+6 \cos (2 \pi 60 t)) \\
\sin (2 \pi 90 t) \\
\boldsymbol{n}(t)
\end{array}\right]
$$

where four components of $\mathbf{s}(t)$ are modulating signals, and one component $\mathbf{n}(t)$ is a random source uniformly distributed in $[-1,+1]$.
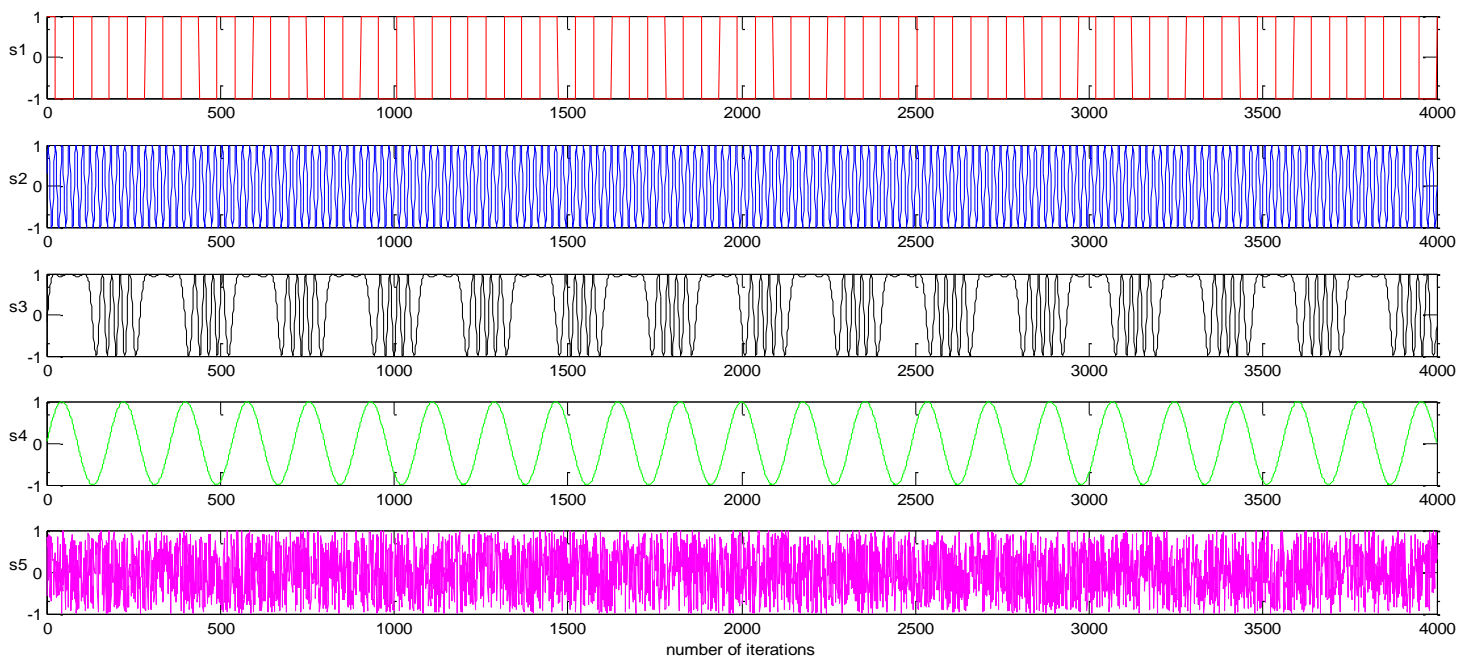

Fig. 4. Source signals.

In the simulation, we sample the mixture $\mathbf{x}(t)=\mathbf{A s}(t)$ with the rate of $10 \mathrm{KHz}$.

We consider $g(\mathbf{y})$ as a prior for the sources. The nonlinearity corresponding to this prior assumption is $g(\mathbf{y})=y^{3}$. The cross-talking error is used to measure the performance of the algorithms and is defined 
as

$$
P I=\sum_{i=1}^{n}\left(\sum_{j=1}^{n} \frac{\left|g_{i j}\right|}{\max _{k}\left|g_{i k}\right|}-1\right)+\sum_{j=1}^{n}\left(\sum_{i=1}^{n} \frac{\left|g_{i j}\right|}{\max _{j}\left|g_{k j}\right|}-1\right)
$$

where $G=W A=\left(g_{i j}\right)$.

In order to compare the performances of different learning rate rules, we use the conventional EASI algorithm with a fixed learning rate, adaptive gradient variable step-size EASI algorithm and this paper's FNN-based learning rate EASI algorithm to separate source signals from the observed mixtures. According to many experiments, select the following optimal experience. When applying the fixed rate algorithms, $\mu=0.08$ was taken, while $\rho=1.5 \times 10^{-5}, \mu_{\min }=0.002$ and $\mu_{\max }=0.018$ were chosen in the adaptive gradient variable step-size EASI algorithm. In this paper's FNN-based learning rate EASI algorithm, we choose $\mu=0.08, \alpha=1.2, \beta=1.8, k_{d}=0.003, k_{0}=600$ and $f(x)=0.14 x^{1.36}$. Via these parameters, the conventional EASI algorithm with a fixed learning rate, adaptive gradient variable step-size EASI algorithm and this paper's FNN-based learning rate EASI algorithm obtained the best results.
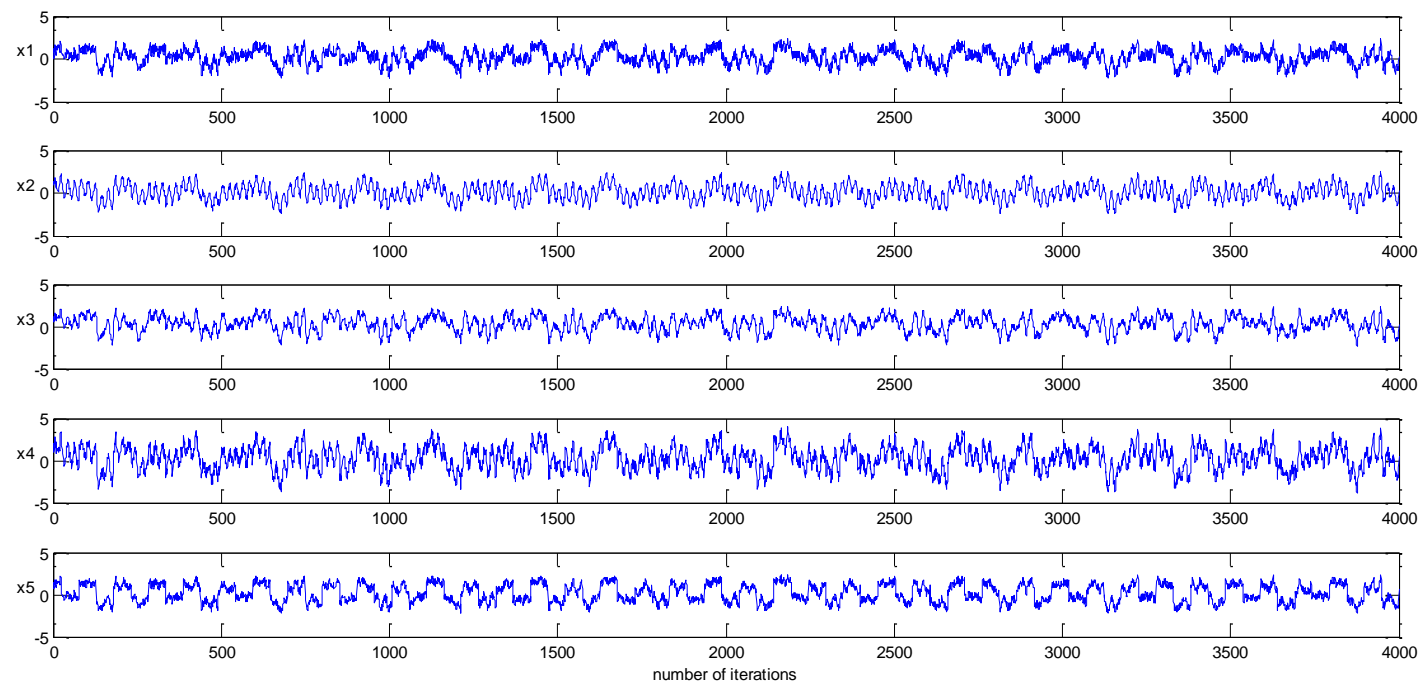

Fig. 5. Mixed signals.

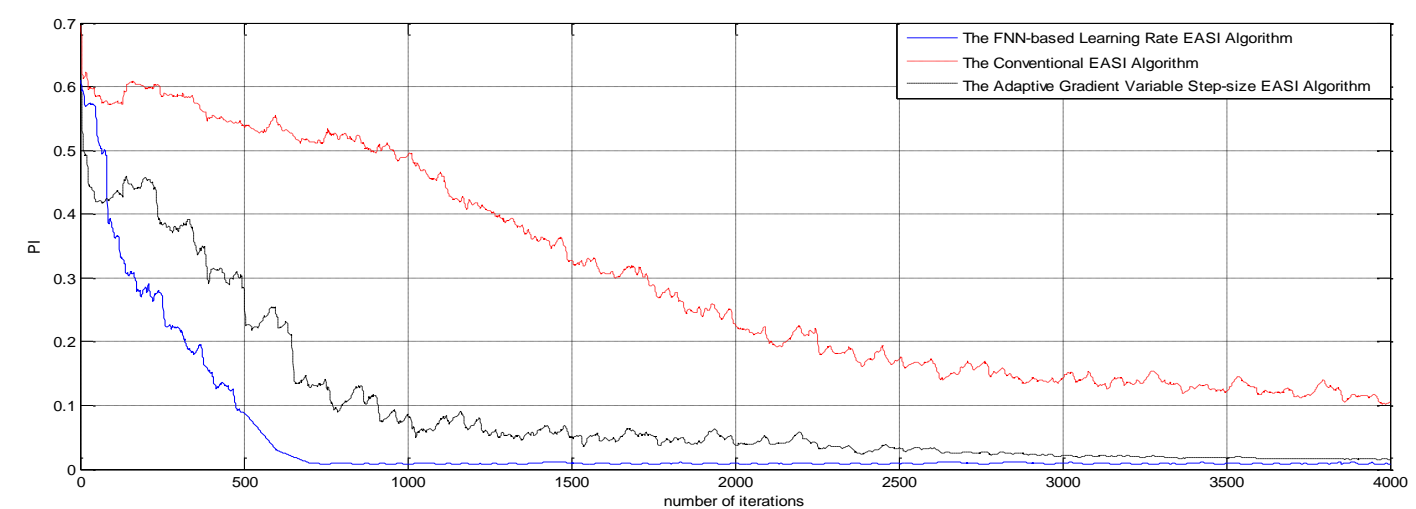

Fig. 6. Mean of cross-talking errors in 200 independent runs for the three methods.

To learn more about performance of these algorithms under different environmental conditions, the paper simulate three kinds of different situations.

(i) The environment experience is stable in the mixing matrix and the elements of the mixing matrix $\mathbf{A}$ are 
random numbers distributed uniformly in $[-1,+1]$.

(ii) The environment experience small continuing changes in the mixing matrix and this is simulated by defining the mixing matrix recursively by

$$
\mathbf{A}(k+1)= \begin{cases}\mathbf{A}(k) & 0 \leq k<4000 \\ \mathbf{A}(k)+\Delta \mathbf{A}(k) & 4000 \leq k \leq 8000\end{cases}
$$

where $a_{i j}(k) \sim U(-1,1), \Delta a_{i j}(k) \sim N(0,0.001)$.

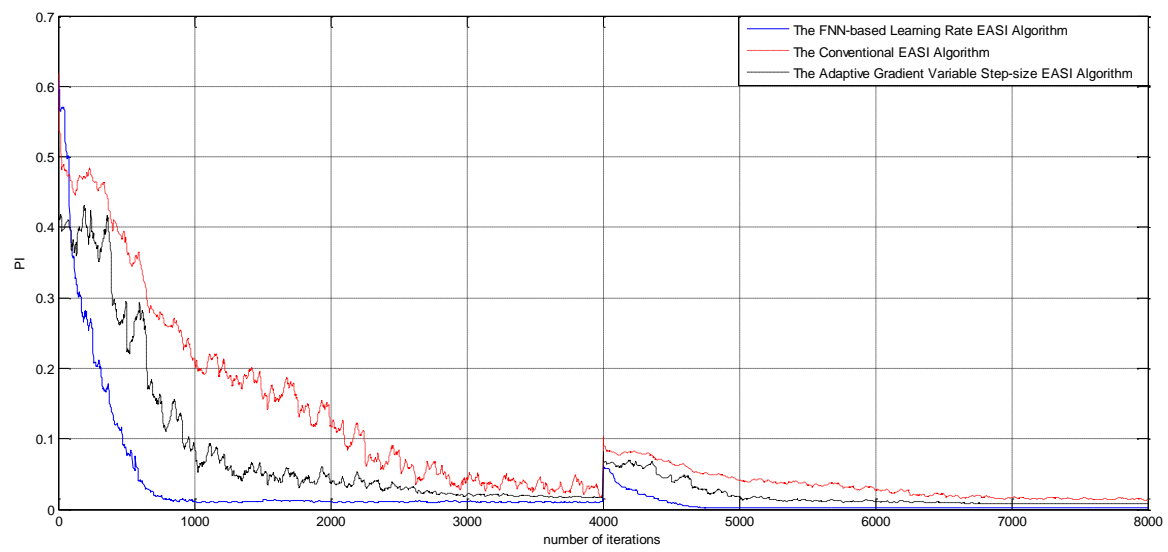

Fig. 7. The mixing matrix drifts for $4000 \leq k \leq 8000$. Mean of cross-talking errors in 200 independent runs for the three methods.

(iii) The environment experience changes abruptly in the mixing matrix and this is simulated by defining the mixing matrix recursively by

$$
\mathbf{A}(k+1)= \begin{cases}\mathbf{A}(k) & 1 \leq k \leq 4000 \\ \theta_{1}^{2} \tilde{\mathbf{A}} & 4001 \leq k \leq 8000 \\ \theta_{2}^{2} \tilde{\mathbf{A}} & 8001 \leq k \leq 12000\end{cases}
$$

where the scalar factor $\theta_{1}, \theta_{2}$ and the coefficients $a_{i j}$ are uniformly distributed in $[0,1]$ and the coefficients $\tilde{a}_{i j}$ are Gaussian distributed. i.e., $\theta_{1,2} \sim U(0,1), a_{i j} \sim U(-1,1), \tilde{a}_{i j} \sim N(0,1)$.

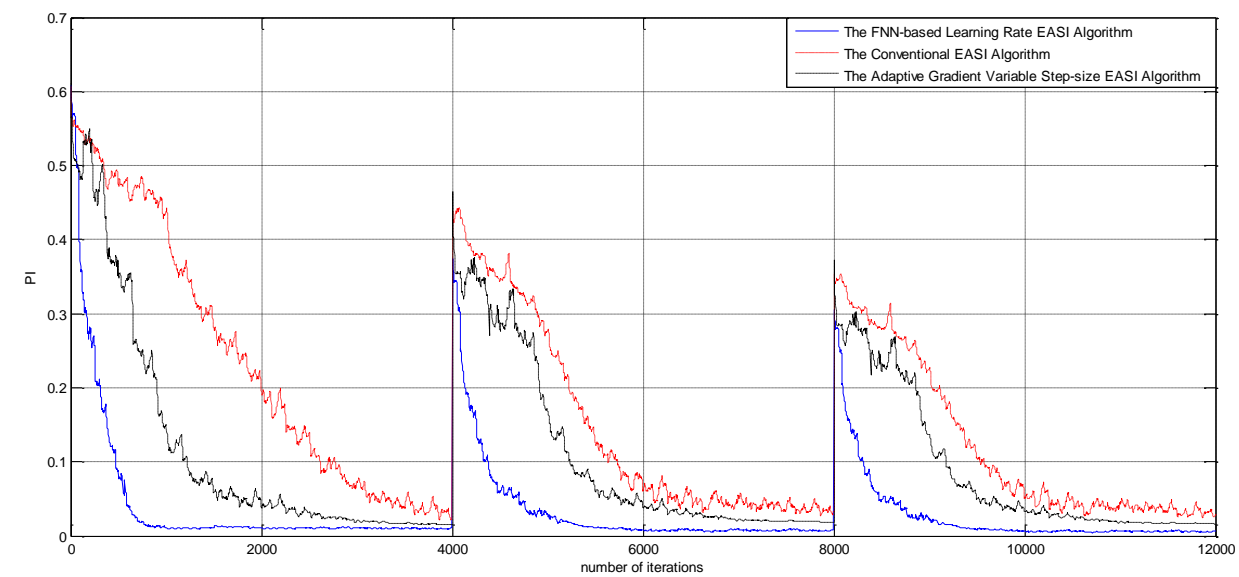

Fig. 8. The mixing matrix changes abruptly every 4000 samples. Mean of cross-talking errors in 200 independent runs for the three methods. 
Based on the above experimental facts, other experiments are used to analyze performance of the proposed algorithm in the stationary environment.

Using the signal to noise ratio (SNR / $d B$ ) as a performance index compares the above algorithm.

$$
S N R=10 \lg \frac{\sum_{k} s_{i}{ }^{2}(k)}{\sum_{k}\left(s_{i}(k)-y_{i}(k)\right)^{2}}(d B)
$$

Table 2. Statistical Results of Four Source Signals

\begin{tabular}{ccccc}
\hline & $\begin{array}{c}\text { Cross-talking } \\
\text { Error(Mean) }\end{array}$ & $\begin{array}{c}\text { Cross-talking } \\
\text { Error(Std.) }\end{array}$ & $\begin{array}{c}\text { Run time } \\
\text { (Avg.) }\end{array}$ & $\begin{array}{c}\text { SNR } \\
(\mathrm{dB})\end{array}$ \\
\hline The conventional EASI algorithm & 0.140 & 0.020 & $0.204 \mathrm{~s}$ & 30.3422 \\
\hline The adaptive EASI algorithm & 0.042 & 0.013 & $0.527 \mathrm{~s}$ & 31.6823 \\
\hline The paper proposed algorithm & 0.014 & 0.002 & $4.349 \mathrm{~s}$ & 33.1627 \\
\hline
\end{tabular}

Fig. 6 shows the resulting from the application of the three methods for 200 trials, which illustrates that the convergence speed of this paper's FNN-based learning rate EASI algorithm is considerably improved upon the two methods. In addition, we can see the new algorithm's misadjustment error in steady state is the least from figure 6.Under non-stationary environment, the step-size reacts immediately to change and its rapid increase allows the algorithm to quickly adjust the demixing $\mathbf{W}$, in turn, reducing the error level. After the adjustment, the step-size again continues to decrease until the next abrupt change as depicted in Fig. 7 and Fig. 8.

\subsection{Ten Source Signals Separation}

Table 3. Statistical Results of Ten Source Signals

\begin{tabular}{ccccc}
\hline & $\begin{array}{c}\text { Cross-talking } \\
\text { Error(Mean) }\end{array}$ & $\begin{array}{c}\text { Cross-talking } \\
\text { Error(Std) }\end{array}$ & $\begin{array}{c}\text { Run time } \\
\text { (Avg.) }\end{array}$ & $\begin{array}{c}\text { SNR } \\
(\mathrm{dB})\end{array}$ \\
\hline The conventional EASI algorithm & 0.178 & 0.022 & $0.404 \mathrm{~s}$ & 20.4121 \\
\hline The adaptive EASI algorithm & 0.092 & 0.011 & $0.627 \mathrm{~s}$ & 26.9823 \\
\hline The paper proposed algorithm & 0.024 & 0.003 & $7.349 \mathrm{~s}$ & 32.0447 \\
\hline
\end{tabular}

For further testing of the robustness of the proposed method, ten source signals were selected and mixed to be separated. The ten source signals were defined as the following:

$$
\boldsymbol{s}(t)=\left[\begin{array}{l}
\operatorname{sgn}(\cos (2 \pi 155 t)) \\
\sin (2 \pi 800 t) \\
\sin (2 \pi 300 t+6 \cos (2 \pi 60 t)) \\
\sin (2 \pi 90 t) \\
\cos (\tan (2 \pi 100 t)) \\
\sin c(2 \pi 100 t) \\
\cos (2 \pi 100 t+5 \sin (2 \pi 0.5 t)) \\
\sin (2 \pi 25 t) \sin (2 \pi 800 t) \\
\cos (\sin (2 \pi 100 t)) \\
n(t)
\end{array}\right]
$$

The separation problem of ten source signals is more difficult than the four source signal, and the results are not as good as for the four source signals separation. Table 3 presents the means and standard deviations of 100 runs. As the separation results are similar to four source signals separation, lower cross-talking error correspond to better separating results, they are not presented. The proposed method exhibits faster and 
more stable convergence while separating ten source signals than the other approaches, which perform without speed and stability. The experimental results can be seen in Table 3.

\section{Conclusions}

The inherent contradiction of the classic EASI algorithm makes it very difficult to balance convergence speed and the steady-state misadjustment error. In this paper, an effective adaptive step-size method to solve the problem is proposed. The separate degree is introduced into Fuzzy Neural Network for solving dynamic problems, and the new algorithm has such property that step-size automatically improves in the beginning of iteration and step-size automatically decreases in the steady-state. Comparing with any other fixed-size or variable step-size EASI algorithm, the new algorithm has faster convergence speed and less steady-state misadjustment error. Extensive simulations have shown that the self-adjusting step-size algorithm is capable of tracking a changing environment and verify the new algorithm may be extended to other EASI algorithm.

\section{Acknowledgment}

Project supported by National Natural Science Foundation of China (Grant No. 61371062); Scientific Research Project for the Returned Overseas Chinese Scholars of Shanxi Province(Grant No. 2013-032); International Cooperation Project of Shanxi Province (Grant No. 2012081031); International Cooperation Project of Shanxi Province (Grant No. 2014081029-01).

\section{References}

[1] Comon, P. (1994). Independent component analysis, a new concept. Signal Processing, 36(3), 287-314.

[2] Hyvärinen, A., \& Oja, E. (2000). Independent component analysis: Algorithms and applications. Neural networks, 13(4), 411-430.

[3] Amari, S., Cichocki, A., \& Yang, H. H. (1996). A new learning algorithm for blind signal separation. Advances In Neural Information Processing Systems, 12(2-5), 757-763.

[4] Leen, T. W., Girolami, M., \& Sejnowski, T. J. (1999). Independent component analysis using an extended infromax algorithm for mixed sub-Gaussian and super-Gaussian sources. Neural Computations, 11, 409-433.

[5] Cardoso, J. F., \& Laheld, B. H. (1996). Equivariant adaptive source separation. IEEE Transactions on Signal Processing, 44(12), 3017-3030.

[6] Aliyu, M. L., Alkassim, M. A., \& Salman, M. S. (2015). A p-norm variable step-size LMS algorithm for sparse system identification. Signal, Image and Video Processing, 9(7), 1559-1565.

[7] Cichocki, A., \& Amari, S. (2002). Adaptive blind signal and image processing: Learning algorithms and applications. John Wiley \& Sons.

[8] Li, G., \& Xu, S. (2006). Adaptive step-size EASI algorithm based on separating degree. Proceedings of IET International Conference on Wireless, Mobile and Multimedia Networks. (pp. 1-4).

[9] Dedieu, J. P., Malajovich, G., \& Shub, M. (2013). Adaptive step-size selection for homotopy methods to solve polynomial equations. IMA Journal of Numerical Analysis, 33(1), 1-29.

[10] Lou, S. T., \& Zhang, X. D. (2003). Fuzzy-based learning rate determination for blind source separation. IEEE Transactions on Fuzzy Systems, 11(3), 375-383.

[11] Tang, X., Zhang, X., \& Ye, J. (2014). Adaptive step-size natural gradient ICA algorithm with weighted orthogonalization. Circuits, Systems, and Signal Processing, 33(1), 211-221.

[12] Kim, E., \& Lee, H. (2000). New approaches to relaxed quadratic stability condition of fuzzy control systems. IEEE Transactions on Fuzzy Systems, 8(5), 523-534.

[13] Mayyas, K., \& Momani, F. (2011). An LMS adaptive algorithm with a new step-size control equation. 
Journal of the Franklin Institute, 348(4), 589-605.

[14] Huang, H. C., \& Lee, J. (2012).A new variable step-size NLMS algorithm and its performance analysis. IEEE Transactions on Signal Processing, 60(4), 2055-2060.

[15] Jiang, Y., Qahouq, J. A. A., \& Haskew, T. A. (2013). Adaptive step size with adaptive-perturbation-frequency digital MPPT controller for a single-sensor photovoltaic solar system. IEEE Transactions on Power Electronics, 28(7), 3195-3205.

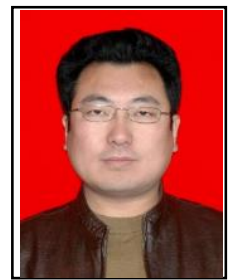

Deng-Ao Li is a Professor at College of Information Engineering in Taiyuan University of Technology (TYUT), Shanxi, China. So far, He has hosted more than ten projects, such as the General Program of the Natural Science Foundation of China, Shanxi Province Science and Technology Research Project, Science Fund for Young Scholars of Shanxi Province, etc. He has published more than forty papers, and five articles are indexed in SCI and fifteen in EI. He edited four textbooks, one of which is the National Eleventh Five-Year Plan textbooks. There are more than twenty patents were opened.

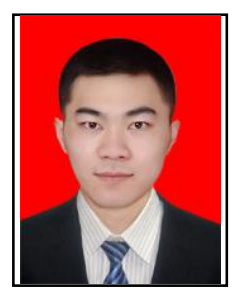

Yan-Fei Bai received a bachelor in information technology from Xinjiang University in 2009 and an MSc in information technology from Taiyuan University of Technology (TYUT) in 2013. His research focus on the study of the ECG signal based on the blind source separation algorithm.

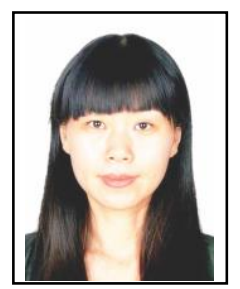

Ju-Min Zhao is a Professor at College of Information Engineering in Taiyuan University of Technology (TYUT), Shanxi, China. She is a Master Instructor in college of information engineering of TYUT. His current research fields include signal and information processing and wireless sensor network. 